
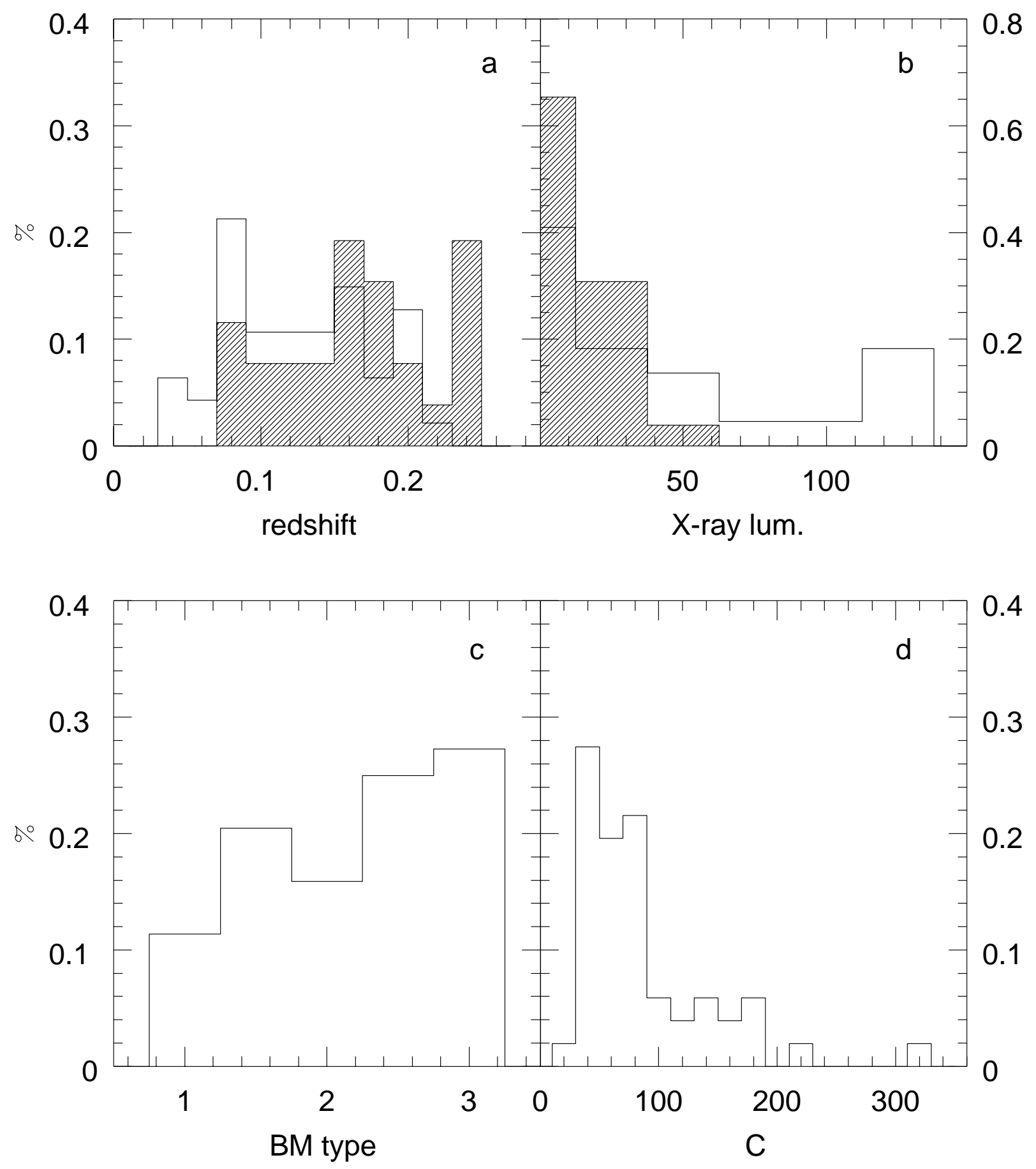
TABLE 1

The SAmple

\begin{tabular}{|c|c|c|c|c|c|c|}
\hline Name & $\alpha$ & $\delta$ & $z$ & $\mathrm{~B}-\mathrm{M}$ & $\mathrm{C}$ & $\mathrm{L}_{X}$ \\
\hline $\mathrm{MS} 0002+1556$ & 00:02:00 & $15: 56$ & 0.116 & $\cdots$ & $\ldots$ & 11.66 \\
\hline $\mathrm{A} 7$ & 00:09:06 & $32: 08$ & 0.107 & II-III & 55 & $\cdots$ \\
\hline $\mathrm{MS} 0011+0837$ & $00: 11: 47$ & $08: 37$ & 0.163 & $\ldots$ & $\ldots$ & 15.27 \\
\hline MS0013+1558 & $00: 13: 27$ & $15: 58$ & 0.083 & $\cdots$ & $\cdots$ & 3.11 \\
\hline MS0026+0725 & $00: 26: 29$ & $07: 25$ & 0.170 & $\cdots$ & $\cdots$ & 6.76 \\
\hline $\mathrm{MS} 0037+2917(\mathrm{~A} 77)$ & $00: 37: 54$ & $29: 15$ & 0.072 & $\mathrm{I}$ & 50 & 15.25 \\
\hline A84 & $00: 39: 12$ & $21: 08$ & 0.103 & II & 76 & 17.82 \\
\hline A115 & $00: 53: 18$ & $26: 03$ & 0.197 & III & 174 & 107.43 \\
\hline MS0102+3255 & 01:02:20 & $32: 55$ & 0.080 & $\cdots$ & $\cdots$ & 2.37 \\
\hline A150 & 01:06:36 & $12: 54$ & 0.060 & I-II & 55 & 2.04 \\
\hline MS0109+3910 & 01:09:24 & $39: 10$ & 0.208 & $\cdots$ & $\cdots$ & 10.41 \\
\hline A175 & $01: 16: 54$ & $14: 37$ & 0.130 & III & 84 & $\cdots$ \\
\hline A180 & $01: 19: 18$ & $02: 45$ & 0.135 & $\mathrm{I}$ & 33 & $\ldots$ \\
\hline A 272 & $01: 52: 24$ & $33: 42$ & 0.088 & III & 52 & $\cdots$ \\
\hline A279 & $01: 53: 48$ & 00:49 & 0.080 & I-II & 70 & $\cdots$ \\
\hline MS0159+0330 (A293) & $01: 59: 24$ & $03: 32$ & 0.163 & II & 87 & 10.53 \\
\hline A318 & 02:10:00 & $26: 12$ & $0.132 *$ & III & 78 & $\cdots$ \\
\hline A376 & $02: 42: 42$ & $36: 39$ & 0.049 & I-II & 36 & 7.24 \\
\hline A399 & $02: 55: 12$ & $12: 49$ & 0.072 & I-II & 57 & 17.93 \\
\hline A401 & $02: 56: 12$ & $13: 23$ & 0.075 & $\mathrm{I}$ & 90 & 43.70 \\
\hline A403 & $02: 56: 12$ & $03: 18$ & 0.103 & II-III & 100 & $\cdots$ \\
\hline A410 & 03:01:18 & $03: 36$ & 0.090 & II & 70 & $\cdots$ \\
\hline $\mathrm{MS} 0301+1516$ & 03:01:44 & $16: 16$ & 0.083 & $\cdots$ & $\ldots$ & 0.64 \\
\hline A439 & $03: 27: 30$ & $24: 37$ & 0.106 & $\cdots$ & 35 & $\cdots$ \\
\hline A468 & $03: 49: 48$ & $21: 16$ & 0.133 & I-II & 34 & $\cdots$ \\
\hline A 478 & $04: 10: 24$ & $10: 21$ & 0.090 & $\cdots$ & 104 & 124.10 \\
\hline MS0433+0957 & $04: 33: 56$ & $09: 57$ & 0.159 & $\cdots$ & $\cdots$ & 17.19 \\
\hline $\mathrm{MS} 0440+0204$ & $04: 40: 31$ & 02:04 & 0.190 & $\cdots$ & $\cdots$ & 18.42 \\
\hline A508 & $04: 43: 18$ & $01: 55$ & 0.148 & III & 85 & $\cdots$ \\
\hline A 520 & $04: 51: 42$ & $02: 52$ & 0.203 & III & 186 & $\cdots$ \\
\hline A566 & $06: 59: 48$ & $63: 22$ & 0.098 & II-III & 127 & 18.79 \\
\hline A586 & $07: 29: 06$ & $31: 44$ & 0.171 & I-II & 190 & 65.70 \\
\hline A 587 & $07: 29: 36$ & $39: 33$ & 0.167 & $\cdots$ & 59 & $\cdots$ \\
\hline A588 & $07: 33: 18$ & $70: 04$ & 0.160 & III & 78 & 2.71 \\
\hline A612 & $07: 57: 48$ & $34: 57$ & $0.153 *$ & II-III & 50 & $\cdots$ \\
\hline A655 & $08: 21: 48$ & $47: 17$ & 0.125 & I-II & 142 & $\cdots$ \\
\hline $\mathrm{A} 665$ & $08: 26: 12$ & $66: 03$ & 0.182 & III & 321 & 124.09 \\
\hline $\mathrm{A} 671$ & $08: 25: 24$ & $30: 35$ & 0.049 & II-III & 38 & 5.79 \\
\hline A733 & $08: 57: 36$ & $55: 49$ & 0.116 & $\mathrm{I}$ & 64 & $\cdots$ \\
\hline MS0904+1651 (A744) & 09:04:30 & $16: 52$ & 0.073 & II & 42 & 3.13 \\
\hline A750 & 09:06:24 & $11: 14$ & 0.162 & III & 142 & $\cdots$ \\
\hline MS0906+1110 & 09:06:34 & $11: 10$ & 0.162 & III & $\cdots$ & 35.23 \\
\hline A910 & 09:59:06 & $67: 24$ & 0.206 & II-III & 222 & 41.95 \\
\hline A923 & 10:03:42 & $26: 09$ & 0.116 & II & 50 & $\cdots$ \\
\hline A963 & 10:14:12 & $39: 16$ & 0.206 & I-II & 134 & 121.43 \\
\hline A1081 & 10:42:00 & $35: 50$ & 0.159 & II-III & 83 & $\cdots$ \\
\hline MS1004+1238 & 10:04:14 & $12: 38$ & 0.166 & $\ldots$ & $\cdots$ & 7.73 \\
\hline MS1050+4946 & $10: 50: 47$ & $49: 46$ & 0.140 & $\cdots$ & $\cdots$ & 11.99 \\
\hline
\end{tabular}


TABLE 1 -Continued

\begin{tabular}{lcccccc}
\hline \multicolumn{1}{c}{ Name } & $\alpha$ & $\delta$ & $z$ & B-M & C & $\mathrm{L}_{X}$ \\
\hline MS1125+4324 & $11: 25: 18$ & $43: 24$ & 0.181 & $\ldots$ & $\ldots$ & 3.34 \\
A1359 & $11: 40: 48$ & $61: 56$ & 0.178 & $\ldots$ & 30 & $\ldots$ \\
A1401 & $11: 49: 30$ & $37: 33$ & 0.165 & III & 153 & $\ldots$ \\
MS1154+4255 & $11: 54: 12$ & $42: 55$ & 0.174 & $\ldots$ & $\ldots$ & 5.39 \\
A1437 & $11: 57: 54$ & $03: 37$ & 0.134 & I-II & 154 & $\ldots$ \\
MS1201+2824 & $12: 01: 31$ & $28: 24$ & 0.167 & $\ldots$ & $\ldots$ & 8.38 \\
A1632 & $12: 50: 30$ & $29: 05$ & 0.196 & II-III & 80 & $\ldots$ \\
MS1253+0456 & $12: 53: 56$ & $04: 56: 15$ & 0.23 & $\ldots$ & $\ldots$ & 16.58 \\
MS1308+3244 & $13: 08: 52$ & $32: 44: 05$ & 0.245 & $\ldots$ & $\ldots$ & 9.79 \\
A1785 & $13: 42: 30$ & $38: 24$ & 0.2136 & I-II & 90 & $\ldots$ \\
A1911 & $14: 22: 24$ & $39: 11$ & 0.191 & II-III & 80 & $\ldots$ \\
MS1401+0437 & $14: 01: 58$ & $04: 37: 21$ & 0.23 & $\ldots$ & $\ldots$ & 4.62 \\
MS1520+3002 & $15: 20: 09$ & $30: 02: 39$ & 0.117 & $\ldots$ & $\ldots$ & 2.30 \\
MS1522+3003 (A2069) & $15: 22: 05$ & $30: 03: 29$ & 0.120 & II-III & 97 & 48.74 \\
MS1531+3118 (A2092) & $15: 31: 18$ & $31: 18: 44$ & 0.067 & II-III & 55 & 2.96 \\
MS1546+1132 & $15: 46: 51$ & $11: 32: 15$ & 0.2260 & $\ldots$ & $\ldots$ & 15.32 \\
MS1558+3321 (A2145) & $15: 58: 24$ & $33: 24$ & 0.088 & $\ldots$ & 48 & 6.63 \\
MS1618+2552 (A2177) & $16: 18: 58$ & $25: 52: 59$ & 0.161 & $\ldots$ & 44 & 8.98 \\
MS2348+2913 & $00: 43: 42$ & $39: 14$ & 0.095 & $\ldots$ & $\ldots$ & 6.39 \\
\hline
\end{tabular}



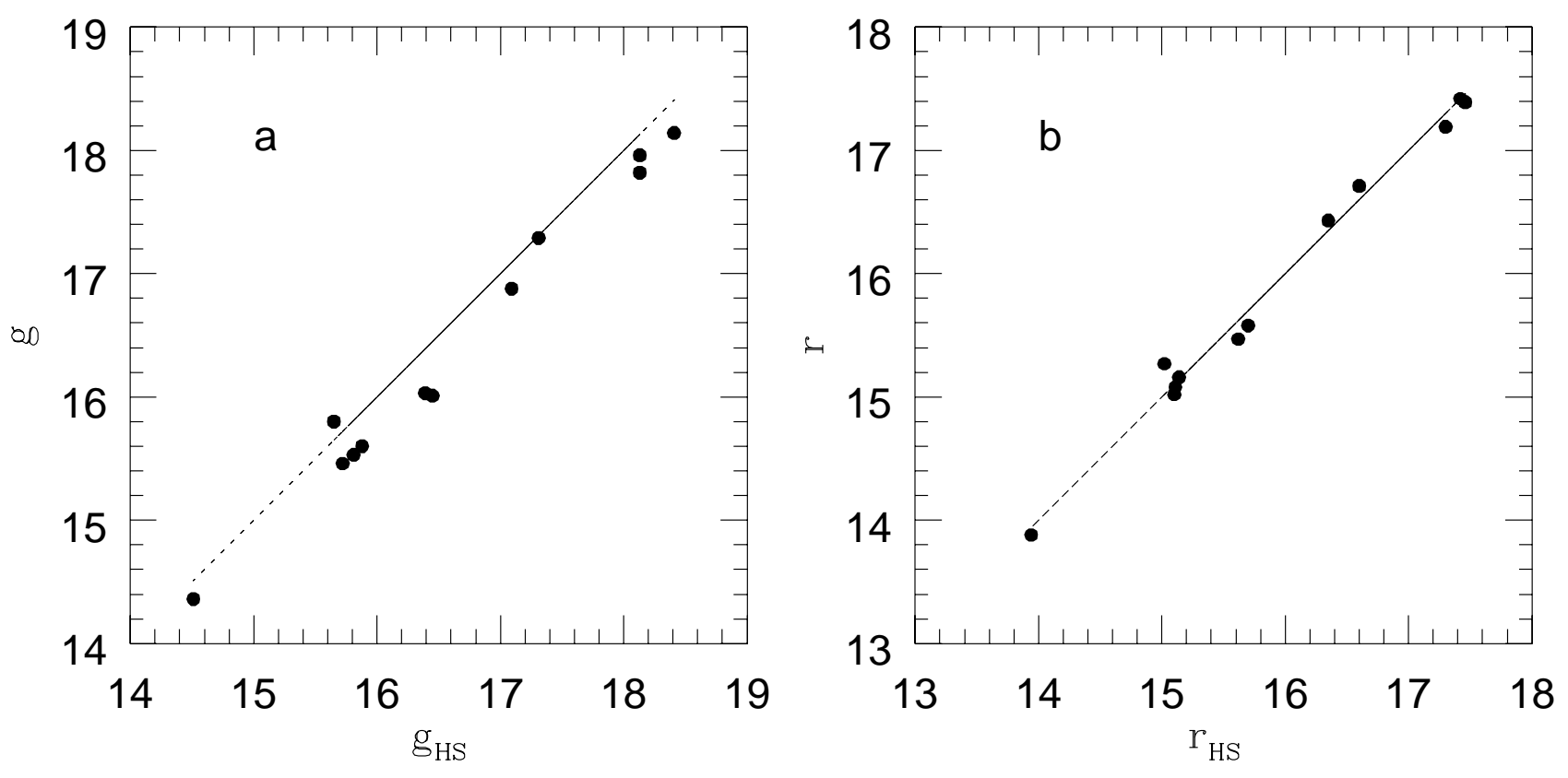
TABLE 2

The Data

\begin{tabular}{|c|c|c|c|c|c|c|c|c|c|c|c|}
\hline \multirow[b]{2}{*}{ Name } & \multirow[b]{2}{*}{$\begin{array}{l}\text { Area } \\
\mathrm{Mpc}^{2}\end{array}$} & \multirow[b]{2}{*}{$\begin{array}{l}\text { No. } \\
\text { gal. }\end{array}$} & \multicolumn{3}{|c|}{$\mathrm{g}$} & \multicolumn{3}{|c|}{$\mathrm{r}$} & \multicolumn{3}{|c|}{$\mathrm{i}$} \\
\hline & & & $\begin{array}{c}\mathrm{t} \\
\mathrm{sec}\end{array}$ & $\begin{array}{l}\text { seeing } \\
\text { arcsec }\end{array}$ & $\mathrm{m}_{l i m}$ & $\begin{array}{c}\mathrm{t} \\
\mathrm{sec}\end{array}$ & $\begin{array}{l}\text { seeing } \\
\text { arcsec }\end{array}$ & $\mathrm{m}_{\text {lim }}$ & $\begin{array}{c}\mathrm{t} \\
\mathrm{sec}\end{array}$ & $\begin{array}{l}\text { seeing } \\
\text { arcsec }\end{array}$ & $\mathrm{m}_{l i m}$ \\
\hline MS0002+1556 & 0.16 & 27 & 1800 & 1.92 & 22.89 & 1200 & 1.74 & 22.88 & 600 & 1.56 & 21.98 \\
\hline $\mathrm{A} 7$ & 0.13 & 31 & 1200 & 1.11 & 22.62 & 1200 & 1.02 & 23.01 & 1200 & 1.02 & 22.65 \\
\hline MS0011+0837 & 0.26 & 17 & 2400 & 2.25 & 22.02 & 1200 & 2.25 & 22.03 & 1200 & 2.25 & 21.85 \\
\hline MS0013+1558 & 0.09 & 17 & 1800 & 2.04 & 22.99 & 1200 & 1.80 & 23.05 & 1200 & 1.83 & 22.51 \\
\hline MS0026+0725 & 0.28 & 28 & 2400 & 1.62 & 22.36 & 1200 & 1.83 & 22.40 & 1200 & 1.20 & 22.07 \\
\hline MS0037+2917 (A77) & 0.07 & 19 & 1200 & 1.44 & 23.32 & 960 & 1.23 & 23.28 & 840 & 1.29 & 22.65 \\
\hline \multirow[t]{2}{*}{ A 84} & 0.91 & 136 & 1800 & 2.73 & 22.94 & 900 & 2.31 & 22.59 & 900 & 2.21 & 22.16 \\
\hline & & & 1800 & 3.28 & 22.94 & 900 & 3.15 & 22.60 & 900 & 2.83 & 22.17 \\
\hline A115 & 1.24 & 121 & 2400 & 1.95 & 23.41 & 1200 & 1.87 & 23.13 & 1200 & 1.46 & 22.87 \\
\hline MS0102+3255 & 0.08 & 29 & 1800 & 2.16 & 23.14 & 1200 & 2.01 & 23.01 & 1200 & 1.89 & 22.47 \\
\hline A150 & 0.61 & 64 & 1200 & 1.82 & 23.20 & 600 & 1.53 & 22.85 & 600 & 1.51 & 22.38 \\
\hline MS0109+3910 & 0.38 & 40 & 3000 & 2.01 & 22.45 & 1500 & 1.89 & 22.48 & 1500 & 1.98 & 22.10 \\
\hline \multirow[t]{4}{*}{ A175 } & 0.75 & 55 & 2400 & 2.79 & 22.02 & 1200 & 2.61 & 21.92 & 1200 & 2.28 & 21.54 \\
\hline & & & 600 & 3.33 & 21.09 & 300 & 2.64 & 21.01 & 300 & 3.12 & 20.72 \\
\hline & & & 2400 & 3.60 & 22.05 & 1200 & 3.36 & 22.01 & 1200 & 3.09 & 21.69 \\
\hline & & & 1200 & 2.43 & 21.60 & 1200 & 1.98 & 21.78 & 1200 & 2.64 & 21.33 \\
\hline A180 & 0.70 & 68 & 2000 & 1.79 & 23.42 & 1000 & 1.87 & 23.14 & 1000 & 1.48 & 22.68 \\
\hline \multirow{2}{*}{ A 272} & 0.19 & 46 & 1800 & 2.10 & 21.43 & 1200 & 2.10 & 22.02 & 1200 & 2.10 & 21.72 \\
\hline & & & 1800 & 2.10 & 20.72 & 1200 & 2.10 & 20.81 & 1200 & 2.10 & 20.80 \\
\hline A279 & 0.08 & 23 & 1800 & 1.69 & 21.77 & 1200 & 1.59 & 21.75 & 1200 & 1.61 & 21.68 \\
\hline \multirow[t]{2}{*}{ MS0159+0330 (A293) } & 0.53 & 60 & 1800 & 1.83 & 23.08 & 1200 & 1.95 & 22.96 & 1200 & 1.50 & 22.36 \\
\hline & & & 1800 & 1.86 & 23.01 & 1200 & 1.92 & 23.04 & 3000 & 1.74 & 22.99 \\
\hline A318 & 0.20 & 35 & 2400 & 2.04 & 22.70 & 1800 & 1.74 & 23.15 & 1600 & 1.62 & 22.07 \\
\hline A376 & 0.03 & 13 & 1200 & 2.88 & 22.40 & 840 & 2.88 & 22.35 & 840 & 2.88 & 21.83 \\
\hline A399 & 0.07 & 34 & 1800 & 1.68 & 21.82 & 900 & 1.89 & 22.06 & 900 & 1.89 & 21.79 \\
\hline A401 & 0.07 & 19 & 1800 & 2.40 & 21.68 & 900 & 2.40 & 21.74 & 900 & 2.40 & 21.45 \\
\hline A403 & 0.46 & 99 & 1800 & 3.20 & 22.64 & 900 & 2.76 & 22.37 & 900 & 2.99 & 21.95 \\
\hline $\mathrm{A} 410$ & 0.10 & 23 & 1800 & 1.47 & 22.63 & 1200 & 1.20 & 22.70 & 1200 & 1.50 & 22.23 \\
\hline MS0301+1516 & 0.09 & 19 & 2400 & 2.10 & 22.76 & 1800 & 2.10 & 22.92 & 1800 & 2.10 & 22.39 \\
\hline \multirow[t]{2}{*}{$\mathrm{A} 439 *$} & 0.27 & 39 & 2400 & 1.59 & 23.19 & 1800 & 1.74 & 23.29 & 1800 & 1.38 & 22.74 \\
\hline & & & 1200 & 1.80 & 22.89 & 900 & 1.89 & 22.95 & 900 & 1.83 & 22.42 \\
\hline A468 & 0.19 & 14 & 1800 & 1.77 & 21.67 & 1200 & 1.95 & 22.09 & 1200 & 1.80 & 21.79 \\
\hline $\mathrm{A} 478$ & 0.36 & 60 & 1800 & 3.04 & 22.43 & 900 & 2.83 & 22.28 & 900 & 3.12 & 21.94 \\
\hline MS0433+0957 & 0.25 & 29 & 2400 & 1.74 & 22.35 & 1800 & 1.86 & 22.58 & 1800 & 1.62 & 22.09 \\
\hline MS0440+0204 & 0.33 & 25 & 1200 & 2.04 & 21.95 & 1500 & 1.95 & 22.44 & 1500 & 2.04 & 22.00 \\
\hline $\mathrm{A} 508 *$ & 0.23 & 33 & 1800 & 1.59 & 21.76 & 1200 & 2.25 & 22.14 & 1200 & 1.98 & 21.27 \\
\hline A520 & 0.36 & 46 & 3000 & 1.83 & 22.24 & 1500 & 1.65 & 22.35 & 1500 & 1.80 & 21.95 \\
\hline A566 & 0.42 & 132 & 1800 & 2.42 & 23.05 & 900 & 2.42 & 22.77 & 900 & 2.18 & 22.29 \\
\hline A586 & 0.28 & 67 & 1800 & 1.62 & 22.31 & 1200 & 1.59 & 22.46 & 1200 & 1.41 & 22.12 \\
\hline A587 & 0.27 & 46 & 1200 & 1.98 & 22.75 & 900 & 2.04 & 22.74 & 900 & 2.07 & 22.18 \\
\hline A588 & 0.92 & 81 & 2400 & 1.64 & 23.67 & 1200 & 1.56 & 23.41 & 1200 & 1.61 & 22.89 \\
\hline A612 & 0.24 & 40 & 1800 & 2.52 & 21.66 & 1200 & 2.52 & 21.92 & 1200 & 2.25 & 21.80 \\
\hline A655 & 0.62 & 124 & 2000 & 2.39 & 23.07 & 1000 & 2.34 & 22.75 & 1000 & 2.08 & 22.28 \\
\hline A665 & 1.11 & 166 & 2400 & 1.64 & 23.66 & 1200 & 1.56 & 23.38 & 1200 & 1.69 & 22.89 \\
\hline \multirow[t]{3}{*}{ A 671} & 0.10 & 55 & 1200 & 2.52 & 22.36 & 840 & 2.43 & 22.31 & 840 & 1.80 & 22.89 \\
\hline & & & 1200 & 2.22 & 22.87 & 840 & 2.19 & 22.81 & 840 & 2.28 & 22.26 \\
\hline & & & 1200 & 1.65 & 22.91 & 840 & 1.62 & 22.76 & 840 & 1.71 & 22.26 \\
\hline A733 & 0.31 & 51 & 2400 & 1.92 & 22.46 & 1200 & 1.56 & 22.46 & 1200 & 1.38 & 22.06 \\
\hline
\end{tabular}


TABLE 2-Continued

\begin{tabular}{|c|c|c|c|c|c|c|c|c|c|c|c|}
\hline \multirow[b]{2}{*}{ Name } & \multirow[b]{2}{*}{$\begin{array}{l}\text { Area } \\
\mathrm{Mpc}^{2}\end{array}$} & \multirow[b]{2}{*}{$\begin{array}{l}\text { No. } \\
\text { gal. }\end{array}$} & \multicolumn{3}{|c|}{$\mathrm{g}$} & \multicolumn{3}{|c|}{$\mathrm{r}$} & \multicolumn{3}{|c|}{$\mathrm{i}$} \\
\hline & & & $\begin{array}{c}\mathrm{t} \\
\mathrm{sec}\end{array}$ & $\begin{array}{l}\text { seeing } \\
\text { arcsec }\end{array}$ & $\mathrm{m}_{\text {lim }}$ & $\begin{array}{c}\mathrm{t} \\
\mathrm{sec}\end{array}$ & $\begin{array}{l}\text { seeing } \\
\text { arcsec }\end{array}$ & $\mathrm{m}_{l i m}$ & $\begin{array}{c}\mathrm{t} \\
\mathrm{sec}\end{array}$ & $\begin{array}{l}\text { seeing } \\
\text { arcsec }\end{array}$ & $\mathrm{m}_{l i m}$ \\
\hline & & & 2400 & 1.74 & 22.61 & 1200 & 1.59 & 22.52 & 1200 & 1.47 & 22.10 \\
\hline MS0904+1651 (A744) & 0.26 & 22 & 1800 & 2.55 & 21.91 & 900 & 2.34 & 21.83 & 900 & 2.22 & 21.49 \\
\hline \multirow[t]{2}{*}{ A750 } & 0.52 & 58 & 2400 & 2.04 & 22.97 & 1800 & 1.95 & 22.83 & 1800 & 1.92 & 22.55 \\
\hline & & & 2400 & 2.28 & 22.83 & 1800 & 2.28 & 22.83 & 1800 & 2.22 & 22.51 \\
\hline MS0906+1110 & 0.26 & 37 & 2400 & 2.31 & 22.77 & 1800 & 2.34 & 22.77 & 1800 & 2.34 & 22.41 \\
\hline A910 & 1.32 & 121 & 3600 & 1.79 & 23.93 & 1800 & 1.79 & 23.61 & 1800 & 1.79 & 23.00 \\
\hline A923 & 0.55 & 86 & 1400 & 1.95 & 23.00 & 800 & 1.69 & 23.04 & 800 & 1.64 & 22.66 \\
\hline A963 & 0.37 & 48 & 1800 & 1.80 & 22.45 & 1200 & 1.80 & 22.49 & 1200 & 1.80 & 22.16 \\
\hline A1081 & 0.25 & 13 & 1800 & 1.65 & 22.25 & 1200 & 2.16 & 22.33 & 1200 & 1.50 & 21.80 \\
\hline MS1004+1238* & 0.27 & 25 & 2400 & 2.10 & 22.49 & 1800 & 2.10 & 22.53 & 1800 & 2.10 & 22.54 \\
\hline MS1050+4946 & 0.21 & 16 & 1800 & 2.34 & 22.63 & 1200 & 2.25 & 22.68 & 1200 & 2.10 & 22.24 \\
\hline MS1125+4324 & 1.10 & 67 & 3200 & 1.95 & 23.59 & 1600 & 2.05 & 23.16 & 1600 & 2.13 & 22.56 \\
\hline $\mathrm{A} 1359 *$ & 0.30 & 17 & 1800 & 1.50 & 22.43 & 1200 & 1.89 & 22.25 & 1200 & 2.01 & 21.59 \\
\hline $\mathrm{A} 1401 *$ & 0.27 & 31 & 1800 & 1.80 & 22.61 & 1200 & 1.80 & 22.17 & 1200 & 1.80 & 22.12 \\
\hline MS1154+4255 & 0.29 & 11 & 2400 & 2.10 & 23.11 & 1800 & 2.10 & 23.17 & 1800 & 2.10 & 21.99 \\
\hline A1437 & 0.70 & 82 & 1800 & 1.87 & 22.38 & 900 & 2.03 & 22.41 & 900 & 1.85 & 22.15 \\
\hline MS1201+2824 & 0.27 & 37 & 2400 & 2.76 & 22.56 & 1800 & 2.70 & 22.57 & 1800 & 2.10 & 21.57 \\
\hline A1632 & 0.35 & 28 & 1200 & 1.44 & 22.67 & 900 & 1.53 & 22.65 & 900 & 1.38 & 21.92 \\
\hline MS1253+0456 & 1.54 & 95 & 1200 & 1.33 & 22.89 & 600 & 1.43 & 22.79 & 600 & 1.38 & 22.34 \\
\hline MS1308+3244 & 1.68 & 47 & 3600 & 2.37 & 22.82 & 1800 & 1.85 & 22.75 & 1800 & 2.55 & 22.29 \\
\hline \multirow{4}{*}{$\mathrm{A} 1785 *$} & 1.57 & 171 & 2100 & 1.50 & 22.88 & 1500 & 1.74 & 22.92 & 1500 & 1.47 & 22.34 \\
\hline & & & 2100 & 1.32 & 23.62 & 1500 & 1.32 & 23.52 & 1500 & 1.35 & 22.92 \\
\hline & & & 2100 & 1.35 & 23.16 & 1500 & 1.35 & 23.00 & 1500 & 1.35 & 22.81 \\
\hline & & & 2100 & 1.41 & 23.54 & 1500 & 1.44 & 23.48 & 1500 & 1.35 & 22.87 \\
\hline $\mathrm{A} 1911 *$ & 0.34 & 23 & 1800 & 1.32 & 22.41 & 1200 & 2.04 & 22.05 & 1200 & 1.50 & 21.45 \\
\hline MS1401+0437 & 1.54 & 62 & 3600 & 1.38 & 23.78 & 1800 & 1.35 & 23.60 & 1800 & 1.25 & 23.15 \\
\hline MS1520+3002 & 1.47 & 105 & 3000 & 1.12 & 24.01 & 1500 & 1.12 & 23.97 & 1500 & 1.12 & 23.53 \\
\hline MS1522+3003 (A2069) & 0.58 & 82 & 1800 & 2.47 & 22.80 & 900 & 2.31 & 22.63 & 900 & 2.65 & 22.07 \\
\hline MS1531+3118 (A2092) & 0.22 & 54 & 1200 & 1.25 & 23.19 & 600 & 1.35 & 23.17 & 600 & 1.22 & 22.63 \\
\hline $\operatorname{MS} 1546+1132 *$ & 0.42 & 21 & 1500 & 1.92 & 21.88 & 900 & 1.89 & 21.87 & 1200 & 2.64 & 20.95 \\
\hline MS1558+3321 (A2145) & 0.10 & 36 & 1200 & 1.50 & 22.26 & 840 & 1.71 & 22.29 & 840 & 1.47 & 21.70 \\
\hline MS1618+2552 (A2177) & 0.93 & 117 & 1800 & 1.35 & 23.46 & 900 & 1.33 & 23.36 & 900 & 1.35 & 22.71 \\
\hline MS2348+2913 & 0.40 & 80 & 2400 & 2.55 & 22.90 & 1200 & 2.46 & 22.66 & 1200 & 2.34 & 22.24 \\
\hline
\end{tabular}



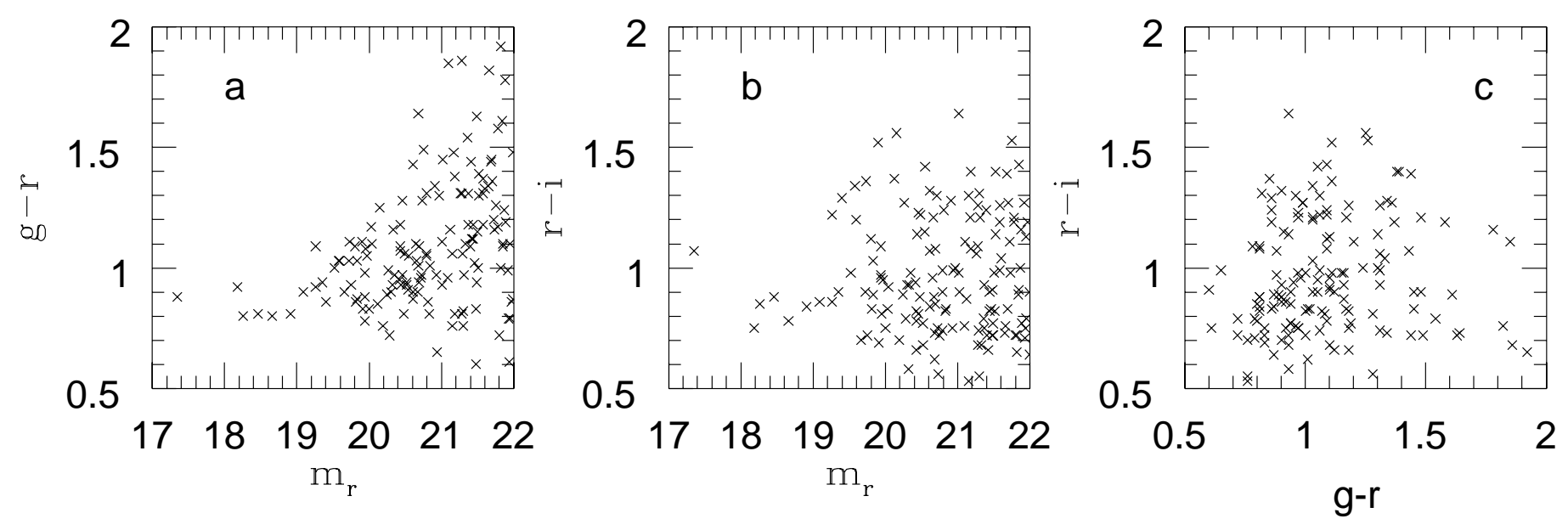


\title{
PHOTOMETRIC PROPERTIES OF CLUSTERS OF GALAXIES
}

\author{
Bianca Garilli, Dario Bottini and Dario Maccagni \\ Istituto di Fisica Cosmica del CNR, via Bassini 15, 20133 Milano, Italy \\ Luis Carrasco ${ }^{1}$ and Elsa Recillas ${ }^{1}$ \\ Instituto Nacional de Astrofisica Óptica y Electrónica, Apartado Postal 51 y 216, 72000 \\ Puebla, Pue., Mexico
}

Received _ ; accepted _

To appear in ApJ Supplements

\footnotetext{
${ }^{1}$ Also Instituto de Astronomia, UNAM, Mexico D.F., Mexico
} 


\begin{abstract}
We report the first results of a long term program aimed at investigating the photometric properties of the cores of Abell and X-ray selected (EMSS, Gioia et al. 1990) clusters of galaxies. We observed 77 clusters of galaxies in the redshift range $0.05 \leq z \leq 0.25$ in the Gunn $g, r$ and $i$ filters: in this paper we present the data on 59 clusters with good absolute photometry and on another group of 8 clusters with acceptable relative photometry. For all these clusters we show color-magnitude diagrams in the two colors: when, as in most cases, the early type galaxy sequence is identifiable, we compare it with the expectation from the Virgo c-m relation (Visvanathan \& Sandage 1977) and find that the Virgo relation holds to $z \sim 0.2$. We do not find any sign of active evolution in cluster galaxies since that epoch, nor in the percentage of blue galaxies or in the early type galaxy colors, if we accept that the scatter is of the order of $0.2-0.3 \mathrm{mag}$ with respect to the expectations on the basis of standard $\mathrm{k}$-corrections. We point out the presence of a certain number of anomalously red galaxies in the $r-i$ color, which are too red with respect to their $g-r$ color to be normal field galaxies at a redshift higher than the cluster one. Finally we briefly compare a few properties of the two subsamples of optically selected and X-ray selected clusters.
\end{abstract}

Subject headings: galaxies, clusters — galaxies, colors 


\section{Introduction}

The fact that large numbers of coeval galaxies can be found only in clusters enhances the importance of these objects as tools in cosmological studies, at the same time providing the substantial database needed to investigate galaxy evolution in a controlled environment. Galaxy clusters can be catalogued and observed up to $z \sim 1$, thus providing a reasonable time span over which the main observable galaxy properties can be compared.

Up to the end of the '70s, clusters of galaxies were optically selected via the galaxy density contrast over the field (Abell 1958, Zwicky et al. 1960-68, Abell, Corwin \& Olowin 1989, ACO). This favors the selection of rich compact clusters, both at low and high redshifts. The Extended Medium Sensitivity Survey (EMSS, Gioia et al. 1990), based on the Einstein Observatory data, has proved that complete catalogues of clusters of galaxies can be obtained by detecting the X-ray emission of the hot intergalactic medium. In the X-rays, cluster visibility is based on the effective presence of a potential well in which the hot gas is confined and on the physical properties of this gas (temperature and density). The X-ray properties of clusters have been widely studied (e.g., Bahcall 1981, Edge \& Stewart, 1991), and their X-ray properties correlated with optical properties, to seek a correspondence between properties of the gas (e.g. temperature, luminosity) and properties of the luminous matter (galaxy density, distribution, etc.). In principle the two selection methods are capable of detecting the same class of objects, as shown by a number of Abell clusters found in the EMSS, in practice, however, several biases are introduced, depending on morphology, richness, and the scatter in the relations between optical and X-ray properties. It is well known (Scaramella et al. 1991) that even the ACO catalog is incomplete for $z$ approaching 0.2 and it is biased toward rich objects. Samples drawn from both optically selected and X-ray selected catalogs could minimize the overall biases and contain clusters of galaxies spanning the whole range of cluster masses and morphologies, 
from which to derive the properties of the luminous matter and the forms of its aggregation. At present, mixed samples with reasonable numbers of clusters can be constructed only up to $z<0.2-0.3$, which corresponds approximately to the look back time when evolutionary phenomena, like the Butcher-Oemler effect (Butcher \& Oemler 1978, Butcher \& Oemler 1984) become detectable.

To fully understand evolutionary phenomena, it is essential to have a solid and statistically significant knowledge of the properties of clusters and of their galaxies for the local universe, where by local we mean a volume where we can reasonably think that galaxies have undergone only passive evolution, i.e. where the Butcher-Oemler effect is negligible. Spectroscopic observations are needed to study the cluster dynamics and to have a better membership probability, but to reach fainter magnitudes, even using a multislit spectrograph, a large amount of time with medium size $(2-4 \mathrm{~m})$ telescopes is needed. On the other hand, by limiting the photometry to the cluster core for reasonably nearby clusters (in a redshift range from $z \sim 0.05$ to $z \sim 0.2$ ) the contamination by non cluster galaxies is expected to be less than $10 \%$ and can be accounted for statistically.

In this paper we present the multicolor photometric data of a sample of clusters which follows the rationale outlined above. We first illustrate the sample composition, observations and data reduction procedures and establish the photometric accuracy. Then we show the color-magnitude diagrams which are then used to isolate the blue and red galaxies in the cluster cores.

Throughout this paper we adopt $H_{0}=50 \mathrm{~km} \mathrm{~s}^{-1} \mathrm{Mpc}^{-1}$ and $q_{0}=0.5$.

\section{The Data}




\subsection{The Sample}

The sample we observed comprises: (a) 39 Abell clusters; (b) 21 EMSS clusters; (c) 7 EMSS clusters which are also included in the ACO catalog. ACO and EMSS clusters have been selected in such a way to mantain a similar redshift distribution. Furthermore, Abell clusters cover all morphological types (according to the Bautz-Morgan classification as reported in the ACO catalog) and richness classes. We secured the redshifts for two clusters previously at unknown distance (spectroscopy of galaxies in the cluster sample will be presented in another paper). We are thus able to present data on 67 clusters with measured redshift.

Figure 1 a to $d$ gives a pictorial representation of the known properties of our cluster sample: the redshift distributions of the 39 Abell clusters and of the 21+7 EMSS clusters; the X-ray luminosity distributions of the 17 Abell clusters observed by the Einstein Observatory and of the 21+7 EMSS clusters; the Bautz-Morgan type and the richness (as defined by the $\mathrm{C}$ parameter in $\mathrm{ACO}$ ) distributions of the Abell clusters. The X-ray luminosity of the Abell clusters has been computed from the IPC count rate, as obtained from the Einstein Observatory Catalog of IPC X-Ray Sources, assuming a Raymond-Smith spectrum with a temperature of $6 \mathrm{keV}$, as it was done in the EMSS.

Table 1 reports this same information in unbinned form for all the 67 clusters in the sample. Column 1 gives the cluster name (either the Abell number or the EMSS truncated source coordinates or both), columns 2 and 3 give the catalog cluster center coordinates for the epoch 1950.0, column 4 gives the redshift: the two new redshifts so far unpublished are marked with an asterisk, while redshifts for the other Abell and EMSS clusters are taken from Abell, Corwin \& Olowin 1989 and from Stocke et al. 1991 respectively; columns 5 and 6 the Bautz-Morgan type and the number of galaxies between $m_{3}$ and $m_{3}+2$ as reported in $\mathrm{ACO}$ (the $\mathrm{C}$ parameter) respectively, and finally in column 7 we give the $\mathrm{X}$-ray luminosity, 
in units of $10^{+43} \mathrm{ergs} \mathrm{s}^{-1}$.

\subsection{The Observations}

The cluster multicolor photometry was carried out in the years 1990-1994 at the 2.1m telescope of the Mexican National Astronomical Observatory at San Pedro Martir, equipped with a CCD of $384 \times 57623 \mu$ pixels, resulting in a field of view of $\sim 2 \times 3$ arcmin. Since December 1993, a new 1024x1024 $22 \mu$ pixels CCD has been available, thus 12 clusters have been observed with a larger field of view $(\sim 4 \times 4 \operatorname{arcmin})$. For 10 clusters we made two or more pointings, to explore a larger area. The total number of nights allocated to this program has been 34, 18 of which proved to be photometric. All clusters have been observed through the three $g, r$ and $i$ Gunn filters with typical exposure times between 20 minutes and 1 hour per filter. When the total exposure time was greater than 20 minutes per filter, the observation was splitted into 2 or 3 exposures, to reduce cosmic ray contamination. The average seeing was $\sim 2$ arcsec.

Standard stars in the Gunn filters (taken from the lists of Thuan \& Gunn 1976 and Wade et al. 1979) were observed all through the nights between cluster exposures to derive flux calibrations. During photometric nights, the accuracy in the zero point is of the order of 0.05 mag. Some clusters were observed in conditions which turned out to be non-photometric. In these cases shorter exposures on the same target were successively made in good weather conditions, and images have been calibrated by matching the magnitudes of stars or compact galaxies in the field.

The journal of the observations is given in Table 2 , for the 67 clusters in the sample. In

column 1, the cluster name is given (an asterisk marks clusters observed in non-photometric conditions), in column 2 the total area explored in $\mathrm{Mpc}^{2}$ at the cluster redshift, in column 3 
the number of galaxies detected in all three filters. Columns 4 to 15 contain the information pertaining to each filter separately: filter id, the exposure time in seconds, seeing in arcsec, and the limiting magnitude. Such limiting magnitude has been computed for each image, and represents the fainter apparent magnitude a pointlike source can have to be detected with a signal to noise ratio of three. The images generally allow to detect objects as faint as $22.5 r$ mag in all the three filters.

\subsection{Data Reduction}

Data reduction was performed in a standard way: after the usual bias subtraction, flat fielding and cosmic ray removal, images in the same filter have been registered and summed.

By careful inspection of the images, we produced lists of objects for each filter. These lists were complemented with the ones produced by the MIDAS/INVENTORY search algorithm run with a low detection threshold and crosschecked among the three independent images. Only objects detected in all the three filters were retained in each cluster catalog. As a second step we rejected all objects which were below any of the limiting magnitudes reported in Table 2. This procedure does not produce clear-cut magnitude limited catalogs, because the effective limits are dependent on the colors of the objects, but exposure times were such that normal ellipticals of $M_{r}=-18$ are generally included in the catalogs of galaxy clusters at $z=0.2$.

Finally, bright stars have been removed from the catalogs. Convolution of a Point Spread Function, representative of our images, with exponential and de Vaucouleurs' galaxy profiles have shown that, beyond the complexity of the two parameter dependence, galaxies could be separated from stars if the objects were brighter than $m_{r} \sim 21$ (Pocar 1992). Thus bright stars were removed from the catalogs on the basis of their FWHM. We recall that in 
the field, stars are a factor 2.5 less numerous than galaxies at $m_{r}=22$ (see e.g. Jarvis \& Tyson 1981).

Two kinds of magnitudes have been computed for each galaxy: an apparent aperture magnitude, and a metric magnitude. Metric apertures are computed within $10 \mathrm{kpc}$ radius. Magnitudes, and errors, have been computed using the IRAF package apphot. In deriving galaxy magnitudes and colors, we have considered both seeing effects and the presence of nearby objects. To include most of the signal in the aperture, angular diameters have been chosen to be twice as large as the largest seeing FWHM in the three filters. In the cases when the aperture magnitude is strongly contaminated by nearby objects, the $10 \mathrm{kpc}$ magnitude has been used. When both magnitudes were affected by nearby objects, the smallest aperture has been used, but a minor weight was given to the point. Magnitudes have been corrected for atmospheric extinction and galactic absorption on the basis of the measured hydrogen column density along the line of sight (Stark et al. 1992), converted to $\mathrm{A}_{g}, \mathrm{~A}_{r}$ and $\mathrm{A}_{i}$ following Schneider, Gunn \& Hoessel 1983. Statistical errors on magnitudes (and colors) depend on the signal to noise ratio,and therefore also on apparent magnitude. In Table 3, we illustrate the statistical accuracy of the color determination as a function of magnitude for the whole sample: $90 \%$ of the galaxies brighter than $\mathrm{m}_{r}=21$ have errors on $g-r$ smaller than 0.068 mag and on $r-i$ smaller than 0.058 mag; $80 \%$ of the galaxies brighter than $\mathrm{m}_{r}=22$ have errors on $g-r$ smaller than 0.09 mag and on $r-i$ smaller than 0.076 mag.

The number of galaxies in each cluster field for which we have multicolor information ranges from 13 to 175 . Colors are available for a total of 3843 galaxies. 


\subsection{Photometric Accuracy}

To check our photometric accuracy, we have compared our magnitudes with those obtained by Hoessel \& Schneider 1985 (HS), who observed 185 Brightest Cluster Galaxies

in the $g$ and $r$ Gunn filters. For this comparison, we use $H_{0}=60 \mathrm{~km} \mathrm{~s}^{-1} \mathrm{Mpc}^{-1}$ and magnitudes within $16 \mathrm{kpc}$ radius apertures, as they did. No galactic extinction was applied, as done by HS. For the 12 galaxies in common with HS, in Figure 2 a and b we plot our magnitudes vs. theirs. The dotted line represents the $y=x$ relation. A linear regression performed on the data of Figures $2 \mathrm{a}$ and $\mathrm{b}$ give results that are compatible with the $y=x$ relation, although a slight shift is present in the $g$ magnitudes, in the sense that $\left\langle g_{\text {thiswork }}>=<g_{H S}>-0.20 \pm 0.14\right.$ (the same relation for the $r$ filter is $\left.<r_{\text {thiswork }}>=<r_{H S}>-0.004 \pm 0.13\right)$. Such discrepancy could be due to the slightly different filter+detector response we have in the $g$ band. We note however that the offset is comparable to the statistical and systematic errors one incurs in when computing magnitudes of large extended objects like the BCGs are. No comparison can be made for the $i$ filter, for lack of other observations.

\section{Results}

\subsection{Color-magnitude Diagrams}

In Figure 3a we show the color magnitude diagrams for all the 59 clusters within our sample observed in optimal photometric conditions, sorted in increasing redshift: $g$ - $r$ and $r-i$ vs. the metric aperture in the $r$ filter. Figure $3 \mathrm{~b}$ shows the same diagrams for the 8 clusters which have been recalibrated. Error bars on magnitude and colors are indicated: they are smaller than the point size for all but the faintest objects. The superimposed dashed line represents the expected color-magnitude relation as derived from Visvanathan 
\& Sandage 1977. The slope of the c-m relation has been computed for our two colors by directly reading from their Figure 2 the values corresponding to the effective wavelengths of our filters + CCD system. We then assumed rest frame colors of $g-r=0.37$ and $r-i=0.31$ for a galaxy of absolute magnitude $M_{r}=-23$. These rest frame colors are the ones obtained by Schneider, Gunn \& Hoessel 1983 in their study of cluster BCMs, once we allow for a 0.1 shift in $g-r$, in order to align our $g$ magnitudes with those of HS. The $\mathrm{k}$-corrections of SGH were used to convert from rest frame colors to the colors expected at the cluster redshifts.

Most clusters show a neat sequence of early type galaxies in both colors. Exceptions are A468 and MS1125+4324 and perhaps MS0013+1558, MS1154+4255 MS1401+0437 and MS1308+3244, to which we must add A439. Lack of an identifiable sequence can occur when the morphological composition is strongly biased toward late types or if the observed region is not the cluster center. This second occurrence is to be excluded in the case of the $\mathrm{X}$-ray selected clusters, since the available coordinates are precise enough to identify the cluster center.

In the following we will consider only the 59 clusters with top quality photometry (Fig. 3a). From the plots, it is clear that: a) the slope of the $\mathrm{c}-\mathrm{m}$ relation describes quite well the data for all clusters with an identifiable sequence. That is to say, the $\mathrm{c}-\mathrm{m}$ effect does not show any evolution since $z \sim 0.2$. b) The adopted colors and absolute magnitude normalization is quite satisfactory for most clusters, but there are cases when one or both colors appear either too blue or too red, beyond any (systematic) photometric error. In Table 4 we list the clusters for which the difference between the "expected" c-m normalization and the observed one differs by more than 0.1 mag in one of the two colors. As shown in Table 3, a shift of 0.1 in color is well out of the statistical errors, much more so if we take into account that galaxies determining the early type sequence are mostly 
brighter than $\mathrm{m}_{r} \sim 20.5$.

It is worth noting that a difference in color of 0.1 cannot be ascribed to errors in the determination of the galactic absorption. For instance, as far as $g-r$ is concerned (this color is much more sensitive than $r-i$ to the interstellar reddening), a 0.1 shift would imply an offset of the same order in $\mathrm{E}(\mathrm{B}-\mathrm{V})$, which translates into $10^{21}$ atoms $\mathrm{cm}^{-2}$ in terms of $N_{H}$. Such values are well above the uncertainties quoted by Burstein \& Heiles 1982 and/or Stark et al. 1992. We have also checked whether discrepancies of this order exist between the measures of Stark et al. 1992 and Burstein \& Heiles 1982. Only in the case of A478 the two measures showed such a large difference, as it is discussed below.

We will now discuss the few cases worth particular notes.

A272. This is a mosaic of two fields, one of which had to be recalibrated. The high dispersion in the sequence is probably due to the lower photometric accuracy.

A478. The early type galaxies in this cluster are much redder than expected, both in $g-r$ and in $r-i$. As noted by Bahcall \& Sargent 1977, this cluster is heavily reddened (they estimate a $E(B-V)=0.7 \pm 0.2)$. The colors of the early type galaxies in this cluster would become as expected from the Virgo $\mathrm{c}-\mathrm{m}$ relation if the hydrogen column density is more than a factor of 3 higher than the value interpolated from the Stark et al. catalog, i.e. if $E(B-V) \sim 0.4$. This is the only case where the value for $E(B-V)$ found in Burstein \& Heiles 1982 survey is significantly different from the Stark et al. 1992 one: 0.24 against 0.1. Even the highest value falls a factor of 2 short of what would be needed to bring A478 colors to "normal". However, the different values in the two catalogs could indicate that the absorption in the direction of A478 is extremely patchy (the closest Stark et al.'s measurement is 0.44 degrees away from the position of the brightest galaxy in A478).

A175. Galaxies in the diagram come from 4 adjacent fields. The brightest galaxy is a 
foreground spiral. The scatter in the sequence is rather large.

A180. The sequence is not so well defined as in other clusters and it might be slightly bluer in $g-r$ than expected.

A612. The redshift of this cluster is based on the spectrum of one galaxy we obtained in November 1994 and should be considered provisional.

A1081. This cluster seems bluer than expected in $g-r$. We would take this fact with caution because the $r$ image has been recalibrated.

A115. The brightest galaxy in the diagram is a foreground elliptical.

On the whole, $\sim 30 \%$ of the clusters have early type sequences which are either bluer or redder than expected on the basis of our normalization of the $\mathrm{c}-\mathrm{m}$ relation. This result could be due to an intrinsic scatter in the colors of early type galaxies belonging to different clusters, similarly to what found by HS for BCMs. On the other hand, within the same cluster such scatter is considerably reduced, as shown by the color-magnitude diagrams. In other words, different clusters evolve in a different way, and this is reflected by the different normalization of the color-magnitude relation. If true, some other difference related to cluster evolution should be present. We have checked if the "bluishness" or "redness" of the c-m relation is somehow tied with other cluster properties, like Bautz-Morgan type, richness, X-ray luminosity or central galaxy density. The low number of objects for which this information is available (16) does not allow us to see any statistically significant correlation. The only trend which can be inferred is with redshift, in the sense that clusters showing a "bluer" c-m relation are at redshift higher than 0.13. An exception to this rule is MS0904+1651 (A744), which is the only nearby cluster having a bluer c-m relation. However, this trend, if real, does not hold in the opposite way, i.e. higher redshift clusters are not always bluer than expected. 


\subsection{Blue and Red Galaxies}

We defined as blue galaxies the ones with a $g-r$ color bluer by 0.3 mag than the early type sequence of the cluster. Clusters have been subdivided into redshift bins of 0.05 , and the percentage of blue galaxies in each bin has been computed over the number of galaxies brighter than $M_{r}=-18$. The percentage of blue galaxies ranges from $\sim 8 \%$ to $\sim 13 \%$, showing an increase with redshift, although with large error bars.

An interesting feature of our color-magnitude diagrams is the presence of a rather large number of red galaxies, defined as those galaxies having both $g-r$ and $r-i$ colors redder than the early-type sequence in each cluster by 0.3 mag. There are 149 such galaxies in our fields, and their color-magnitude diagrams and color-color diagram are shown in Figure 4 . The $g-r$ vs. $m_{r}$ diagram is quite compatible with what expected from field galaxies in the cluster backgrounds (see, e.g., Neuschaefer \& Windhorst 1995): such a diagram would indicate a population of field galaxies which should be at a redshift of about 0.3-0.5. On the other hand, there are more than 50 galaxies occupying the upper part of the color-color diagram. These galaxies have magnitudes $19.5 \leq m_{r} \leq 22$ and have $r-i>1.0$ and $0.8 \leq g-r \leq 2.0$. Normal, high redshift field galaxies are not expected to be found in that part of the diagram. The $r-i$ color would yield a redshift $z>0$. , but their $g-r$ color is not red enough if these galaxies are far away. The anomaly of the spectral energy distribution of these objects results from their emission in the redshifted $i$-band, i.e. above 0.7-0.8 $\mu$. Other authors observed galaxies with similar characteristics in other cluster fields: in a small sample of IR selected clusters, Aragon-Salamanca et al. 1993 find galaxies with extreme infrared colors, unmatched by the observations of field galaxies. In less recent years, Bautz, Loch \& Wilkinson 1982, Couch et al. 1983, Ellis et al. 1985 mentioned the presence of anomalously red galaxies in the clusters they observed. Our observations suggest that the phenomenon is more common and requires redshift determinations to be 
properly assessed.

\subsection{Optical vs. X-ray Selected Clusters}

Our mixed sample contains objects found over the whole sky (the Abell clusters) and objects serendipitously detected over a much smaller and unconnected area (the EMSS clusters). However, the photometric properties of the cores of both classes of objects, from the slope of the color-magnitude diagrams, to the presence of an early-type sequence, to the galaxy number-density gradients, do not allow one to distinguish the origin of the cluster studied. The reasons why the EMSS clusters are not included in the Abell catalog do not depend on the properties, numbers or colors of the galaxies within a few hundred kpc radius of the centre. The cores of clusters are alike, irrespective of the selection method.

\section{Conclusions}

Our two-color photometric survey of a sample of clusters of galaxies ranging in redshift from $z \sim 0.05$ to $z \sim 0.23$ shows that:

(a) the photometric properties of the galaxies in the cores of X-ray selected and optically selected clusters are substantially the same;

(b) the color-magnitude effect found in Virgo generally fits well the data for clusters in the whole redshift range we surveyed, once appropriate k-corrections are applied. There are clusters which deviate by $0.2-0.3 \mathrm{mag}$ from the expectation, either toward the red or the blue. This is of the order of the scatter present in the colors of bright cluster ellipticals. The fainter early type galaxies share the same colors, and follow the Virgo c-m effect with a scatter which can be evaluated to be around 0.1 mag in our colors; 
(c) on average, there is no detectable Butcher-Oemler type evolution of galaxies since $z \sim 0.2$. However, the cluster to cluster scatter of the percentage of blue galaxies in all redshift bins (even normalized to the surveyed area of each cluster) is very large.

(d) in our fields, we found a sizable number of galaxies redder in both colors than the early type sequence. About a third of them have anomalous colors, in the sense that their $r-i$ color is too red to be compatible with their $g-r$ color if they are background field galaxies.

We thank S. Pocar for help in the reduction of some of the data. Discussions with G. Chincarini and his suggestions have been invaluable in assessing several of the points we addressed in this paper. We are grateful to the UNAM-OAN Time Allocation Committee for the generous support given to this program throughout the years. Finally, we warmly acknowledge the assistance received by all the OAN staff both in San Pedro and in Ensenada. 
Table 3. Accuracy of galaxy colors

\begin{tabular}{|c|c|c|c|c|c|c|}
\hline \multirow[b]{2}{*}{$\mathrm{m}_{r}$} & \multicolumn{3}{|c|}{$\sigma_{g-r}$} & \multicolumn{3}{|c|}{$\sigma_{r-i}$} \\
\hline & $\leq 0.05$ & $\leq 0.07$ & $\leq 0.09$ & $\leq 0.05$ & $\leq 0.07$ & $\leq 0.09$ \\
\hline$\leq 21$ & $81 \%$ & $91 \%$ & $95 \%$ & $87 \%$ & $94 \%$ & $97 \%$ \\
\hline$\leq 22$ & $59 \%$ & $71 \%$ & $80 \%$ & $66 \%$ & $77 \%$ & $86 \%$ \\
\hline
\end{tabular}


Table 4. "blue" and "red" clusters

\begin{tabular}{lcccc}
\hline \hline \multicolumn{1}{c}{ Name } & $g-r$ & $\Delta m$ & $r-i$ & $\Delta m$ \\
\hline \multirow{2}{*}{ A2092 } & $\ldots$ & & red & 0.1 \\
A744 & blue & 0.1 & $\ldots$ & $\ldots$ \\
MS0301+1516 & red & 0.2 & $\ldots$ & $\ldots$ \\
A410 & red & 0.2 & $\ldots$ & $\ldots$ \\
A478 & red & 0.2 & red & 0.1 \\
A403 & red & 0.1 & $\ldots$ & $\ldots$ \\
A180 & blue & 0.1 & $\ldots$ & $\ldots$ \\
A612 & blue & 0.1 & $\ldots$ & $\ldots$ \\
A1081 & blue & 0.2 & $\ldots$ & $\ldots$ \\
MS0433+0957 & $\ldots$ & & red & 0.1 \\
A588 & blue & 0.1 & $\ldots$ & $\ldots$ \\
A750 & red & 0.1 & $\ldots$ & $\ldots$ \\
MS0026+0725 & blue & 0.2 & $\ldots$ & $\ldots$ \\
A115 & blue & 0.1 & $\ldots$ & $\ldots$ \\
A520 & blue & 0.2 & blue & 0.1 \\
MS0109+3910 & blue & 0.2 & blue & 0.1 \\
\hline & & & & \\
\hline
\end{tabular}




\section{REFERENCES}

Abell, G.O. 1958, ApJS, 3, 211

Abell, G.O., Corwin, H.G., Jr., \& Olowin, R.P. 1989, ApJS, 70, 1

Aragon-Salamanca, A., Ellis, R.S., Couch, W.J., \& Carter, D. 1993, MNRAS262, 764

Bahcall, N.A. \& Sargent, W.L.W 1977, ApJ, 217, L19

Bahcall, N.A. 1981, ApJ, 247, 787

Bautz,M., Loh, E., \& Wilkinson, D.T. 1982, ApJ, 255, 57

Burstein, D. \& Heiles, C. 1982, AJ, 87, 1165

Butcher, H. \& Oemler, A., Jr. 1978, ApJ, 219, 18

Butcher, H. \& Oemler, A. Jr. 1984, ApJ, 285, 426

Couch, W.J.,Ellis, R.S., Godwin, J., \& Crater, D. 1983, MNRAS, 205, 1287

Edge, A.C. \& Stewart, G. 1991, MNRAS, 252, 428

Ellis, R.S., Couch, W.J., MacLaren, I., \& Koo, D.C. 1985, MNRAS, 217, 239

Gioia, I.M., Maccacaro, T., Schild, R.E., Wolter, A., Stocke, J.T., Morris, S.L., \& Henry, J.P. 1990, ApJS, 72, 567

Hoessel,J. \& Schneider,D. 1985, AJ, 90, 1648

Jarvis, J.F., \& Tyson, J.A. 1981, AJ, 86, 476

Neuschaefer, L.W. \& Windhorst, R.A. 1995, ApJS, 96, 371

Pocar, S. 1993, Thesis, University of Milan 
Scaramella, R., Zamorani, G., Vettolani, G. \& Chincarini, G. 1991, AJ, 101, 342

Schneider, D.P., Gunn, J.E. \& Hoessel, J.H. 1983, ApJ, 264, 337

Stark A. A. et al. 1992, ApJS 79, 77

Stocke, J.T., Morris, S.L., Gioia, I.M., Maccacaro, T., Schild, R.E., Wolter, A., Fleming, T.A. \& Henry, J.P. 1991, ApJS, 76, 813

Thuan, T.X. \& Gunn, J.E. 1976, PASP, 88, 543

Visvanathan, N. \& Sandage, A. 1977, ApJ, 216, 214

Wade, R.A., Hoessel, J.G., Elias, J.H. \& Huchra, J.P. 1979, PASP, 91, 35

Zwicky, F., Herzog, E., Wild, P., Karpowicz, M. \& Kowall, C.T. 1960-68, Catalog of Galaxies and of Clusters of Galaxies, Pasadena: California Institute of Technology 
Fig. 1. - a) redshift distribution of Abell (white) and EMSS (grey) clusters in our sample; b) X-ray Luminosity distribution, symbols as in $a$; c) Morphology distribution of Abell clusters in our sample; d) Richness distribution of Abell clusters in our sample

Fig. 2.- a) our $g$ central magnitudes vs. Hoessel \& Schneider (1985) magnitudes; the dotted line represents the $y=x$ relation; b) as $a$ for the $r$ filter.

Fig. 3.- Color-magnitude diagrams of the clusters in the sample. The dotted line represents the virgo c-m relation (see text). a) the 59 clusters with top quality photometry; b) the 8 clusters which have been recalibrated

Fig. 4.- color magnitude ( $g-r$ (a) and $r-i(\mathrm{~b}))$ and color color diagrams (c) of the 149 galaxies defined as "red" 